\title{
Sleep deprivation affects extinction but not acquisition memory in honeybees
}

\author{
Syed Abid Hussaini, Lisa Bogusch, Tim Landgraf, and Randolf Menzel ${ }^{1}$ \\ Neurobiologie, Instituet fuer Biologie, Freie Universität Berlin, D-14195 Berlin, Germany
}

\begin{abstract}
Sleep-like behavior has been studied in honeybees before, but the relationship between sleep and memory formation has not been explored. Here we describe a new approach to address the question if sleep in bees, like in other animals, improves memory consolidation. Restrained bees were observed by a web camera, and their antennal activities were used as indicators of sleep. We found that the bees sleep more during the dark phase of the day compared with the light phase. Sleep phases were characterized by two distinct patterns of antennal activities: symmetrical activity, more prominent during the dark phase; and asymmetrical activity, more common during the light phase. Sleep-deprived bees showed rebound the following day, confirming effective deprivation of sleep. After appetitive conditioning of the bees to various olfactory stimuli, we observed their sleep. Bees conditioned to odor with sugar reward showed lesser sleep compared with bees that were exposed to either reward alone or air alone. Next, we asked whether sleep deprivation affects memory consolidation. While sleep deprivation had no effect on retention scores after odor acquisition, retention for extinction learning was significantly reduced, indicating that consolidation of extinction memory but not acquisition memory was affected by sleep deprivation.
\end{abstract}

Much of what we know today about sleep is mostly from mammalian models and humans. Although there has been considerable progress in understanding sleep, there is very little known about its function. Of particular importance is the relationship between sleep and memory. Recent findings (Born et al. 2006; Gais et al. 2006; Daurat et al. 2007) have shown that slowwave sleep (SWS, a part of non-REM sleep) is important for the formation of declarative memory (hippocampus-dependent) and that REM sleep is important for the formation of procedural memory (hippocampus-independent). Additionally, declarative but not procedural memory could be boosted when subjects were stimulated with electrical potentials in phase with slow wave activity during the first $45 \mathrm{~min}$ of SWS sleep (Marshall et al. 2006). Another study (Rasch et al. 2007) showed a boost in retention when subjects were exposed to odors (used as context during learning) during SWS sleep. Most data today indicate that sleep mostly affects declarative memories in humans by sparing procedural memories, indicating that sleep is not required for all kinds of memories (Rasch et al. 2007; Wilhelm et al. 2008).

The earliest descriptions of sleep in insects were presented in papers by Fiebrig (1912) and Hoffmann (1937). Later sleep was described in insects like the honeybees (Kaiser and Steiner-Kaiser 1983; Kaiser 1988; Schuppe 1995; Sauer et al. 2003, 2004), fruit flies (Hendricks et al. 2000; Shaw et al. 2000; Greenspan et al. 2001), solitary bees (Kaiser 1995), cockroach (Tobler 1983), pacific beetle cockroach (Stephenson et al. 2007), moth (Andersen 1968), paper wasp (Klein 2003), locust (Schuppe and Burrows 1998), and scorpion (Tobler and Neuner-Jehle 1988) and in other invertebrates like the jellyfish (Seymour et al. 2004) and crayfish (Ramon et al. 2004). Despite early studies on moths (1968), honeybees (1983), and other insects, sleep research in insects was not very active until the discovery of sleep in Drosophila (Hendricks et al. 2000; Shaw et al. 2000), which spurred renewed interest in insect sleep research. There is much more known about Drosophila sleep

'Corresponding author.

E-mail menzel@neurobiologie.fu-berlin.de; fax 49-30-838-5455. Article is online at http://www.learnmem.org/cgi/doi/10.1101//m.1578409. now than in any other insect because of its genetic tractability. Studies in honeybees have shown that these insects sleep like other diurnal insects both inside of the hives (Kaiser 1988; EbanRothschild and Bloch 2008; Klein et al. 2008) and under laboratory conditions (Sauer et al. 2003), and that their sleep is controlled by the interaction of a circadian clock and a sleep homeostat. Like in mammals, sleep-deprived bees sleep longer the following night, a phenomenon called "sleep rebound." This showed that sleep in honeybees is regulated by homeostatic mechanisms (Sauer et al. 2004). Sleep, at least in adult bees, is also influenced by their circadian clock, which drives strong diurnal rest-activity rhythms.

In insects, there are very few studies that link sleep with learning and memory. In Drosophila there is convincing evidence that sleep is regulated by the mushroom bodies (Joiner et al. 2006), structures that are known for their role in learning and memory formation (Heisenberg 2003) and appear to play a dynamic role in promoting sleep (Pitman et al. 2006). Additionally, it has been shown that Drosophila hyperkinetic mutants have reduced sleep and poor memory (Bushey et al. 2007), and long-term courtship memories in flies are abolished by sleep deprivation within $24 \mathrm{~h}$ after training (Ganguly-Fitzgerald et al. 2006). The mechanism of sleep-related learning is becoming clear in Drosophila; scientists have attempted to rescue sleep loss-induced learning by targeting a single neuronal structure (Seugnet et al. 2009).

As far as we know, there is no study in honeybees addressing the relationship between sleep and memory formation. Our aim in the present study was (1) to characterize sleep under conditions that allow appetitive olfactory conditioning (Bitterman et al. 1983) by monitoring the movements of the antenna, as an indicator of sleep (Sauer et al. 2003, 2004), (2) to compare sleep in trained versus naïve bees, and (3) to interfere with sleep to understand whether sleep deprivation affects memory.

\section{Results}

Below are a series of experiments (for experimental design, see Table 1) showing (1) the characterization of sleep by monitoring the "antennal activity," a well-known indicator of sleep; (2) the 
Table 1. Experimental design

\begin{tabular}{|c|c|c|}
\hline Experiment & Recording/conditioning/retrieval & Groups \\
\hline Antennal activity & Antennal activity recording & \\
\hline \multirow[t]{3}{*}{ Sleep rebound } & Day 1: Antennal activity recording & Group 1: SD by shaking \\
\hline & Day 2: Sleep deprivation & Group 2: Non-SD \\
\hline & Day 3: Antennal activity recording & \\
\hline \multirow{5}{*}{$\begin{array}{l}\text { Olfactory conditioning } \\
\text { and antennal activity }\end{array}$} & Day 1: Three-trial absolute & Group 1: CS+US \\
\hline & $\begin{array}{l}\text { conditioning }+ \text { antennal } \\
\text { activity recording }\end{array}$ & \\
\hline & Day 2: One memory retrieval & Group 2: CS-alone \\
\hline & & Group 3: Air-alone \\
\hline & & Group 4: US-alone \\
\hline \multicolumn{3}{|c|}{ Sleep deprivation (SD) and memory retrieval } \\
\hline \multirow[t]{2}{*}{ Acquisition memory } & $\begin{array}{l}\text { Day 1: Three-trial absolute } \\
\text { conditioning + SD }\end{array}$ & Group 1: SD \\
\hline & Day 2: One memory retrieval & Group 2: Non-SD \\
\hline \multirow[t]{3}{*}{ Extinction memory } & $\begin{array}{l}\text { Day 1: Three-trial absolute } \\
\text { conditioning }\end{array}$ & Group 1a: Extinction trials + SD \\
\hline & Day 2: Two extinction trials + SD & Group 1b: Extinction trials + non-SD \\
\hline & Day 3: One memory retrieval & $\begin{array}{l}\text { Group 2a: No extinction trials + SD } \\
\text { Group 2b: No extinction trials + non-SD }\end{array}$ \\
\hline
\end{tabular}

SD indicates sleep deprived.

effect of various conditioning tasks on sleep; and (3) the effects of sleep deprivation on memory.

\section{Antennal activity}

We first characterized antennal movements during the light and dark phase and identified characteristic patterns of flagellal movements (Fig. 1). Each bee was fed with four to six drops of $30 \%$ sucrose solution and placed under the web camera for video recording. Antennal activity was monitored using the tracking program, and the resulting angles were analyzed. Figure 2 shows the position of left (black) and right (gray) antennae over several hours. We observed uncoordinated movements (ellipse), asymmetrical activity (rectangles), and symmetrical activity (arrows) of the antennae described in detail below. Based on a previous definition of rest in Drosophila (Shaw et al. 2000), sleep was defined as immobility of both antennae lasting $5 \mathrm{~min}$ or longer. The amount of sleep (sleep duration in minutes) and number of sleep bouts were calculated for every hour. Cumulative sleep for the entire day (19 h: 12-h dark/7-h light) was also calculated. Additionally, user-defined antennal patterns were identified, and their occurrences were calculated every hour.

Figure 2 shows the angles of both flagellae plotted against time of the day. The position of the right antenna (RA) moved between $0^{\circ}$ (extension) and $100^{\circ}$ (flexion), and that of the left antenna (LA) between $80^{\circ}$ (flexion) and $180^{\circ}$ (extension) relative to the line connecting the scapes. More than $85 \%$ of the bees rested their antennae in flexed positions (RA $100^{\circ}$ and LA $80^{\circ}$ ). Bees slept more during the dark phase than the light phase. After the onset of the dark phase, the hourly average of sleep (sleep duration) (Fig. 3A) increased slowly and peaked around midnight $(P<0.01)$. The number of sleep bouts (Fig. 3B) also increased $(P<$ $0.05)$ during the dark phase.

The angular movements of the right (gray color) and left flagellum (black color) were not completely random but showed distinct synchronous patterns of movements (Fig. 3 C,D). We identified two patterns: symmetrical and asymmetrical activity. During symmetrical activity, both flagella moved from flexion toward extension, i.e., moving in opposite directions to each other (Fig. 3C). During asymmetrical activity, both antennae display parallel scanning movements in the same direction simultaneously (Fig. 3D). Symmetrical activity was significantly more frequent during the dark phase (peak, $6.13 \mathrm{~h}$ ) than during the light phase (peak, 3.42 h) $(P<0.01)$. Asymmetrical activity appeared higher during the light phase (peak, $6.64 \mathrm{~h}$ ) than the dark (peak, $4.67 \mathrm{~h}$ ) phase, but this effect was not statistically significantly different.

\section{Olfactory conditioning and antennal activity}

Using the classical conditioning of proboscis extension response (PER) in honeybees, where an odor (conditioned stimulus [CS]) is reinforced with a sugar water reward (unconditioned stimulus [US]), we next asked whether bees trained to a rewarded odor (CS+US) spent different times sleeping the following night compared with bees that were not trained but received the same amount of CS or US stimulation. On day 1 , bees were either conditioned to an olfactory stimulus or exposed to CS alone, US alone, or air alone. Bees were randomly allotted to one of the four groups: group 1, CS+US; group 2, CS alone; group 3, air alone; and group 4, US alone. Each training or stimulus exposure trial lasted $60 \mathrm{sec}$, and each bee received three trials at 10-min intertrial intervals (ITIs). After $27 \mathrm{sec}$ of air, group 1 was presented with $4 \mathrm{sec}$ of CS and 3 sec of US with a 1-sec overlap (conditioning), group 2 was presented with 4 sec of CS alone, group 3 was presented with 4 sec of air alone, and group 4 was presented with 3 sec of US alone. The stimulus exposure was followed by air until the end of $60 \mathrm{sec}$. One hour after the training, all bees were fed with four to six drops of 30\% sucrose solution. Each bee was placed inside the sleep-box under a web camera, and their antennal activities were recorded. The bees were kept on 12-h light/12-h dark phase at room temperature. On day 2, each group was tested for retention by presenting them with one trial of CS only.

Analysis of antennal activities showed that the cumulative sleep phases differed significantly between the four groups (Fig. 4A). Cumulative sleep was 337.5 min for group 1 (CS+US), 407.44 min for group 2 (CS alone), 436.4 min for group 3 (air alone), and $558.2 \mathrm{~min}$ for group 4 (US alone). Group 1 bees slept significantly less than bees in group $4(P<0.01)$ and group $3(P<0.05)$. Group 2 bees showed a trend toward longer sleeping compared with group 1 bees, but the difference was not statistically significant.

The 24-h retention tests indicated memory for the CS in group 1, while groups 2, 3, and 4 showed low response levels to the CS (Fig. 4B).

\section{Sleep rebound}

Sleep can be prevented by shaking the animals in a vortex for $5 \mathrm{~min}$ at intervals of $5 \mathrm{~min}$ for over $15 \mathrm{~h}$ (Figs. 1, 5A). We asked whether sleep deprivation induced by this form of shaking leads to a rebound of sleep during the following night. Therefore, on day 1 the bees were divided into two groups. Each group was placed under a web camera such that all pairs of flagella could be clearly seen. Their antennal activities were video recorded overnight. On day 2, group 1 bees were sleep deprived, and group 2 bees were not sleep deprived but otherwise treated similarly. On day 3 , both groups were placed again under their respective web cameras for antennal activity recordings.

Bees that were sleep deprived (group 1), showed early sleep onset (not significant) before the start of dark phase and prolonged sleep $(P<0.01)$ after the end of dark phase (Fig. 5A); whereas the nonsleep-deprived bees (group 2) had normal sleep onset and waking periods. 
A
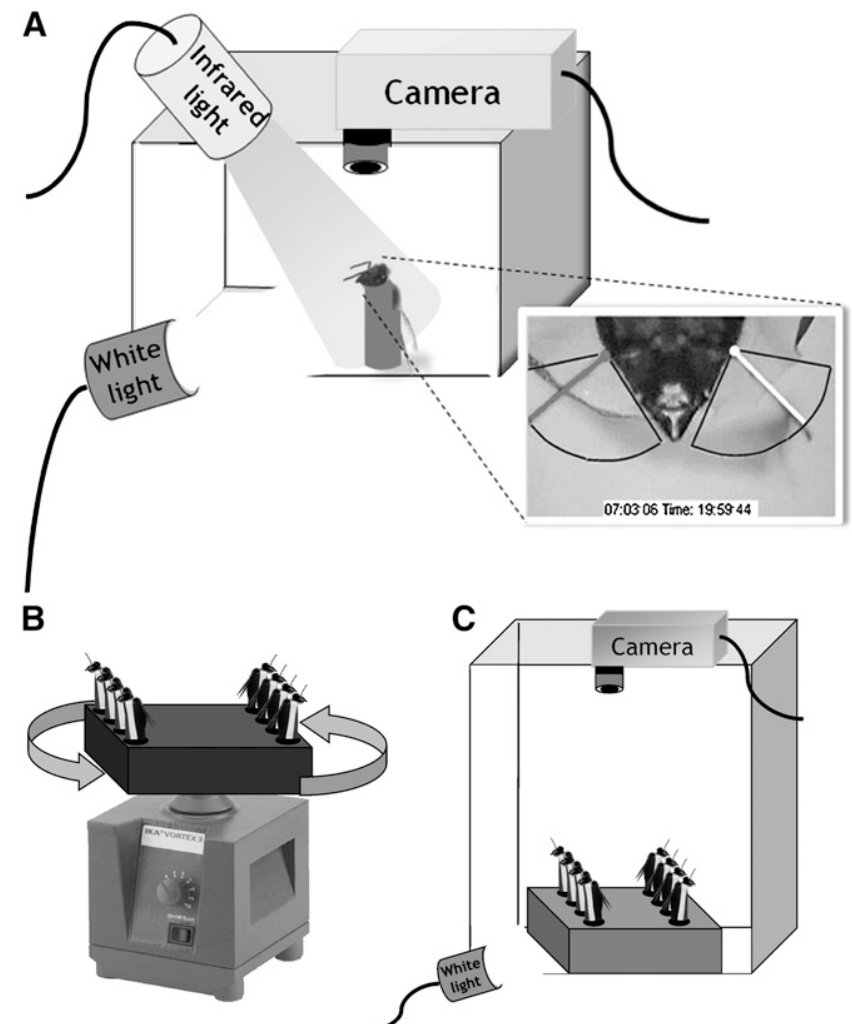

в



Figure 1. (A) Sleep monitoring box: A bee was placed inside a fivesided box under a web camera such that dark antenna were visible against the white background. Infrared light was used for recording in the dark cycle, and white light was used during the light cycle. (Inset) Antenna tracking: The flagella of the antennae were monitored using a custom made program (see Materials and Methods). After the user defined the left (white) and right (gray) flagellum, the program automatically detected their movements and reported their positions in angles. (B) Sleep deprivation by shaking: Bees were placed on the vortex immediately after training and were alternatively shaken (80-120 rpm) and rested for 5 min until the following day. (C) Measuring multiflagella activity: To monitor flagella of many antennae, a larger sleep monitoring box setup was used. Up to eight bees were placed inside the sleep box, and all flagella were simultaneously video recorded with the web camera at $10 \mathrm{fps}$.

\section{Sleep-deprivation and memory consolidation}

\section{Acquisition memory}

We next asked whether memory consolidation requires uninterrupted sleep phases during the night following acquisition odor training. Bees were conditioned with three trials of CS+US with a 10-min ITI and were divided into two groups. Group 1 bees were sleep deprived by placing them on a vortex immediately after conditioning and were shaken at 100-120 rpm every 5 min for over $15 \mathrm{~h}$ under 12-h light/12-h dark phase. Group 2 bees were not sleep deprived and were placed under the same 12-h light/12-h dark phase. On day 2, both groups were tested for memory retention by presenting them with one trial of CS alone (this is also called an extinction trial because the CS is not rewarded and further trials would lead to extinction of memory; see next section). Both groups showed similar acquisition curves (Fig. 5B), and the retention tests on day 2 showed no significant difference ( $P>0.05$; group 1; 64.3\%; group 2, 61.7\%). Thus, sleep deprivation after initial acquisition of odor conditioning did not lead to a change in the 24-h retention scores, indicating either that memory consolidation was not compromised by the interruption of sleep or that the memory is so strong that the reduction of consolidation caused by sleep deprivation did not lead to reduced retention scores.

\section{Extinction memory}

Initial acquisition in odor conditioning leads to very robust memory even after three conditioning trials. The lack of a detrimental effect on memory consolidation by interfering with sleep phases by frequently shaking the animals at regular intervals during the night might be due to saturating processes of memory formation. We, therefore, searched for a way to test memory consolidation of a weaker memory and thus selected extinction learning. Bees learn to reduce their learned responses to a rewardpaired odor by the exposure to the conditioned odor without the reward (CS-only presentation, extinction trial). On day 1 , bees were conditioned as in the experiment before by three trials of CS+US pairing using $10 \mathrm{~min}$ of ITI. These animals were then divided into two groups. On day 2, group 1 bees were presented with two trials of CS alone (extinction trials), and group 2 bees were not presented with any CS (no extinction trials). Group 1 and group 2 bees were further divided into two groups each. Group 1a and group $2 \mathrm{a}$ bees were sleep deprived in the same way as in the experiment before by placing them on a vortex and shaking them for $5 \mathrm{~min}$ at $100-120 \mathrm{rpm}$ every $5 \mathrm{~min}$ for $15-16 \mathrm{~h}$. Group $1 \mathrm{~b}$ and group $2 \mathrm{~b}$ bees were not sleep deprived and were otherwise treated similarly. On day 3 , all bees were tested for retention by presenting them with the CS used for conditioning on day 1.

All four groups learned equally well on day 1 (Fig. 6A,B, left side of the figure). On day 2 , the two groups exposed to two extinction trials also performed the same (Fig. 6A). Groups $2 \mathrm{a}$ and $2 \mathrm{~b}$ were not exposed to any stimulus during day 2 . On day 3 , all groups were subjected to an extinction test by one trial of CS only. Group 1b, which was not exposed to sleep deprivation, had significantly lower retention scores $(P<0.01)$ on day 3 compared with group $1 \mathrm{a}$, which was sleep deprived during the night between day 2 and day 3 (Fig. 6A, right side). The control groups $2 \mathrm{a}$ and $2 \mathrm{~b}$ gave similar retention scores on day 3 , indicating that sleep deprivation interfered with memory consolidation after extinction learning but not after initial acquisition learning.

\section{Discussion}

Sleep in honeybees has been characterized previously by Kaiser (1988) and Sauer et al. (2003) and more recently by two research groups (Eban-Rothschild and Bloch 2008; Klein et al. 2008); however, its relation to memory consolidation has not been studied so far. Since odor conditioning of restrained bees is a well-established paradigm of learning and memory research in the bee (Bitterman et al. 1983; Bicker and Menzel 1989; Menzel 2001; Menzel and Giurfa 2001), we first characterized sleep in such restrained conditions and then performed odor conditioning experiments controlling sleep during the following night. The flexed positions of antennae or antennal immobility were used as indicators for sleep-like behavior. The flexed positions of antennae (LA $80^{\circ}$ and RA $100^{\circ}$ ) were more frequent during the dark phase than during the light phase. The symmetrical and asymmetrical patterns observed here are novel findings and might indicate characteristic features of different sleep phases. The symmetrical activity could result from abrupt interruptions of the balance of muscle tone or from unintentional movements such as rapid eye movements. It is interesting to find that such movements occurred only during the dark phase and thus might indicate a particularly deep sleep phase. Asymmetrical movements, on the other hand, are reminiscent of antennal movements during wake phases, although much slower, and thus might indicate less deep sleep phases. Such an interpretation is supported by the 

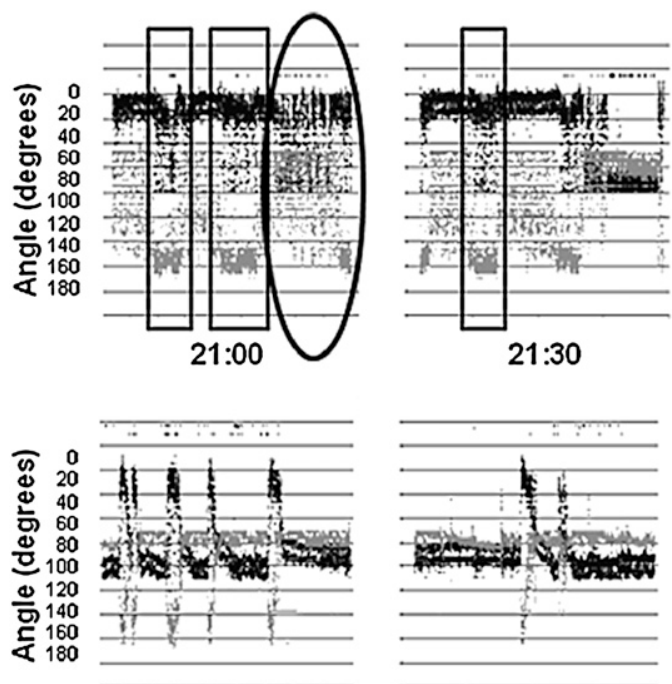

03:00

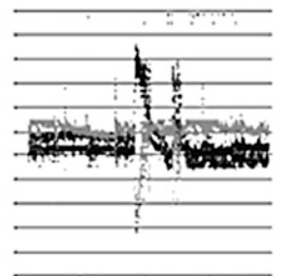

03:30

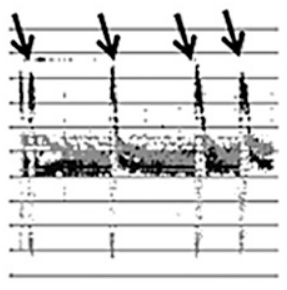

22:00

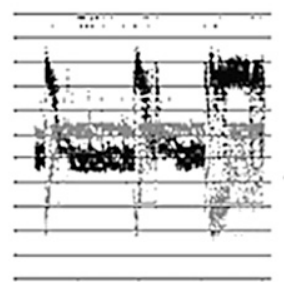

04:00

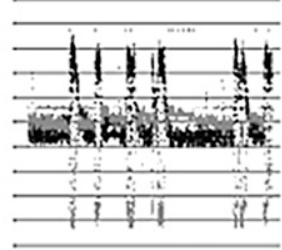

22:30

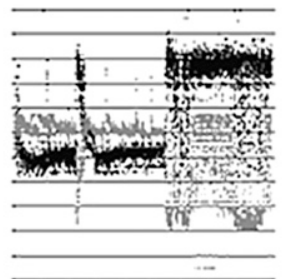

04:30



23:00

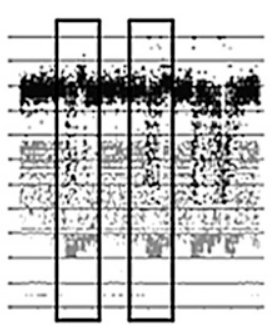

05:00

\section{Time of day}

Figure 2. Antennal activity: The position (in degrees) of left (black) and right (gray) antennae were recorded for 19 h, starting from 15:00-10:00 h. Here we show a few selected hours from the original continuous recording of the activities of each antenna. At 21:00 h, asymmetrical (or scanning) activity is shown in rectangular boxes. Here both antennae are moving in the same direction. Toward the end of 21:30 $\mathrm{h}$, the antennae become immobile for long periods and are interrupted by bouts of symmetrical activity (arrows) where both antennae simultaneously move in opposite direction to each other. Again at 05:00 h, there is asymmetrical activity. Apart from the two activities, bees also showed uncoordinated activity as seen in the later part of 21:00 $\mathrm{h}$ indicated by an ellipse.

finding that they occurred mostly in the light phase and were less frequent during the dark phase.

We found that restrained bees show by their antennal mobility all signs of sleep as characterized before in more natural conditions and spent more time sleeping during the dark phases compared with the light phase. The longest duration of sleep occurred $7 \mathrm{~h}$ after the onset of the dark phase. These results corroborate nicely previous studies by Sauer et al. (2003). Furthermore, bees compensate for interrupted sleep by sleeping longer the following night. Analysis showed that after day 1 the bees slept normally, but the night after sleep deprivation sleep duration was longer in sleep-deprived bees compared with nonsleep-deprived bees. This rebound of sleep has also been shown before (Sauer et al. 2004).

To study the effect of learning on sleep, we conditioned bees with different stimuli and observed their sleep the following night. Group 1, which was conditioned by pairing sucrose reward (US) with an odor (CS) showed reduced sleep compared with other groups (groups 3 and 4). As expected, group 1 bees responded to the CS more strongly than did bees of the other groups. Most importantly, group 4 , which received the same amount of US as those of group 1, slept the most of all four groups. This finding rules out the possibility that the US controls sleep, e.g., making the animals more aroused by feeding them the afternoon before the following night. An appetitive stimulus such as sucrose is known to activate octopaminergic neurons of the ventral unpaired median (VUM) neurons of the subesophageal ganglion (Schroter et al. 2007), and the activity of one of these neurons, the VUMmx1, was found to provide a sufficient reinforcing signal in odor conditioning (Hammer and Menzel 1998). Injection of octopamine (OA) systemically or into the antennal lobe or the mushroom body arouses the animal, leads to more sucrose uptake (Bicker and Menzel 1989), and can act as a substitute for the US if injected locally into the antennal lobe or the mushroom body calyces shortly after an odor CS (Hammer and Menzel 1998).
Furthermore, OA injections increase the antennal scanning in honeybees (Pribbenow and Erber 1996). Taking all these observations together, it would appear reasonable to assume that the US in the conditioned group 1 and the US-only in group 4 should cause the same effect on sleep if the arousal effect of US via an activation of the OA system was responsible for the reduced sleep. This possibility can be ruled out from our data, leaving us with the conclusion that bees that learned an odor a day earlier, slept less not because they were stimulated with the US or being placed in the experimental conditions without stimulation (group 3); instead, they associated the odor with the reward. Interestingly, no statistically significant difference between the conditioned animals and the CS-only animals (group 2) was found, indicating that the experience of a stimulus alone (odor) is also an important parameter. Note that all bees were fed with sugar water (US) $1 \mathrm{~h}$ after the training before monitoring the sleep. It could be that bees presented with CS-only formed an association with the rewarded US $1 \mathrm{~h}$ later. This association was sufficient to reduce sleep but not strong enough to retain the memory of the CS+US association. To study the effect of sleep on memory consolidation, we sleep deprived bees during the night following conditioning. Memory consolidation in bees is known to occur in several ways (Menzel 2001). Short-term memory is consolidated to an amnesia-resistant form of memory within a few minutes after learning. Middle-term memory develops after multiple learning trials within a few hours after learning, and the transition to an early form of long-term memory (translation-dependent form of long-term memory) occurs within $24 \mathrm{~h}$ after learning. This latter form of memory consolidation after several learning trials is the one studied here. Deprivation of sleep by shaking has shown to be effective in honeybees and causes sleep rebound the following day as documented in our study and one by Sauer et al. (2004). Since our sleep deprivation technique used $5 \mathrm{~min}$ of shaking and $5 \mathrm{~min}$ of rest, we cannot rule out the possibility that bees could have slept during the 5-min rest period. However, we believe this to be very unlikely. Also, we 



Figure 3. Sleep is defined as an immobility of the flagella lasting $5 \mathrm{~min}$ or more. (A) Sleep duration is the cumulative time of sleeping per hour. Sleep duration increases after the onset of the dark cycle (dark bar) and peaks around midnight. During this time, bees sleep on average half the time ( $27 \mathrm{~min} / \mathrm{h}$ ). Sleep duration decreases toward the end of the dark cycle. (B) Sleep bouts: Sleep phases are interrupted by symmetrical and asymmetrical movements of the flagella. A sleep bout is defined by these interruptions. The number of sleep bouts increase in the dark cycle and peak around midnight. $\mathrm{N}=73$. (C) Symmetrical activity is the activity in which both flagellae moved in opposite directions to each other. This leads to flagella either moving away from each other (right flagellum moving right and left flagellum moving left) or toward each other (right flagellum moving left and left flagellum moving right). The number of symmetrical movements was calculated every hour. Symmetrical activity was significantly higher $(P<0.01, t$-test $)$ during the dark cycle compared with the light cycle. (D) Asymmetrical activity is the activity in which both flagella moved together in the same direction simultaneously. The number of asymmetrical movements was calculated every hour. Asymmetrical activity appeared higher during the light cycle compared with the dark cycle, but this effect was not significantly different. $N=73$.

found no effect of sleep deprivation in animals that had been conditioned to an odor CS with multiple trials the day before (called here "acquisition learning" to contrast it to "extinction learning"; see below). Retention scores were not different between sleep-deprived and untreated bees (group 1 and group 2). We conclude that memory consolidation after acquisition training to an odor is not compromised by sleep deprivation.

The failure of a deprivation effect on the consolidation of initial odor conditioning either could result from a strong memory trace or could be related to a particular kind of learning. It is known from sleep studies in mammals that sleep (or the lack of sleep) does not affect all kinds of memory (Silvestri 2005; Robertson 2009). Therefore, we chose to target another form of memory called extinction memory. When a previously rewarded odor (CS) is presented without US, a new memory that CS predicts in the absence of reward is formed (extinction memory). The consolidation of extinction memory has been studied intensively in honeybees (Eisenhardt and Menzel 2007). For example, one group (Stollhoff et al. 2005) found that consolidation of extinction memory can be blocked by injecting emetine (a protein synthesis inhibitor) after extinction trials. We subjected two groups (groups 1a and $1 \mathrm{~b}$ ) of bees to three-trial learning on

A of bees in each group. the first day and two extinction trials on the second day. As expected, both groups showed reduced learning after extinction trials, documenting extinction memory. After subjecting group 1a to sleep deprivation, they failed to show the reduced retention scores on second day, while group $1 \mathrm{~b}$ that was not sleep deprived showed reduced retention scores. We used two additional control groups (groups $2 \mathrm{a}$ and $2 \mathrm{~b}$ ) to show that shaking per se does not

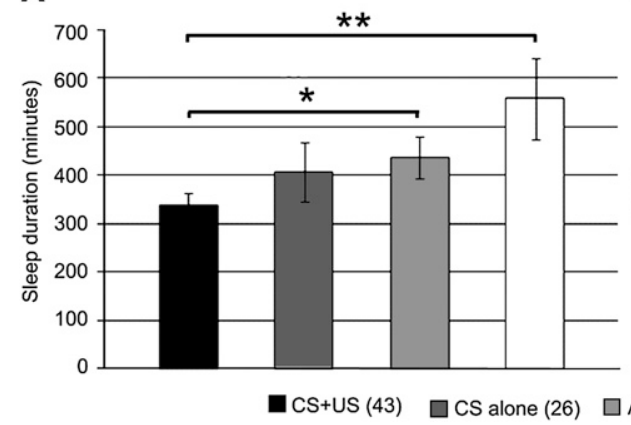

Figure 4. (A) Cumulative sleep during the night following odor conditioning (group 1, CS+US), or stimulus exposure (group 2, CS-only; group3, air-only; group 4, US-only). Bees conditioned with CS+US showed significantly lower cumulative sleep compared with air alone $\left({ }^{*} P<0.05\right)$ and US alone $\left({ }^{* *} P<0.01\right)$ groups using Wilcoxon rank-sum test (or Mann-Whitney $U$-test) (Bonferroni correction, $\alpha$ value set at $P<0.05$ ). The lower cumulative sleep value for the CS+US group was not significantly different from that of the CS alone group. $(B)$ Retention scores $24 \mathrm{~h}$ after conditioning (group 1) or stimulus exposure (groups 2-4). The CS+US group showed the highest retention scores $\left(P<0.01, \chi^{2}\right.$ test), whereas the low PER response values for the other three groups were not significantly different $\left(P>0.05, \chi^{2}\right.$ test) from the spontaneous response levels on day 1 . Numbers in parentheses are number 
A

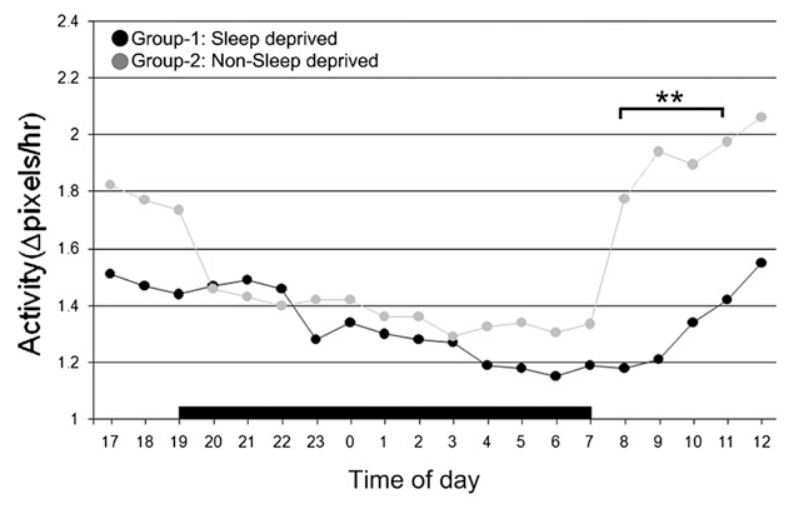

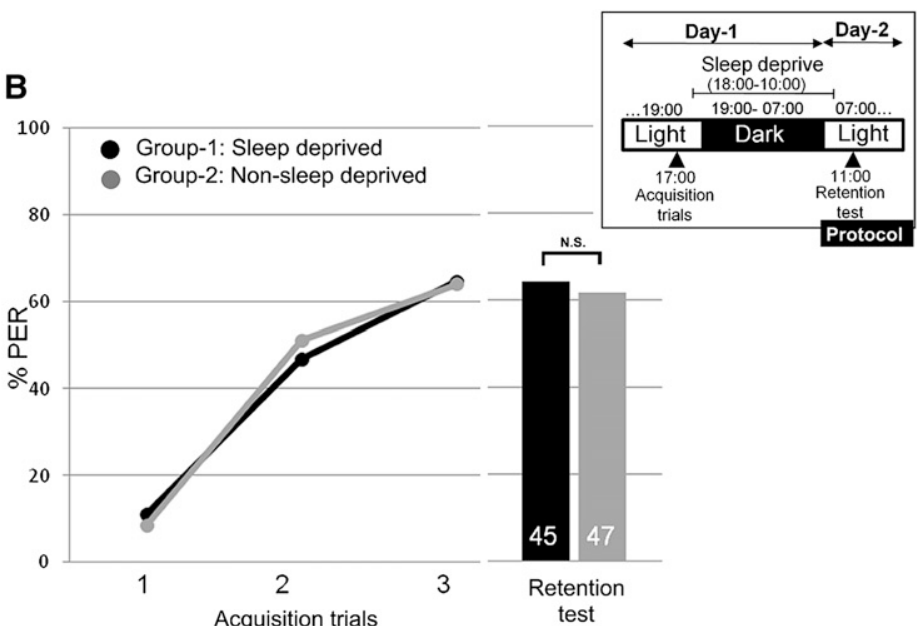

Figure 5. (A) Sleep rebound: Group 1 bees $(\mathrm{N}=23)$ were sleep deprived by shaking and showed reduced antennal activity (prolonged sleep) even after the onset of the light cycle on the following day compared with group 2 bees $(N=19)$, which were not sleep deprived. The reduced activities were significantly different (Wilcoxon signed-rank test with Bonferroni correction, $\alpha$ level set at 0.05) at hours 08:00-11:00 h. (B) Sleep deprivation after acquisition learning: On day 1, two groups of bees (group 1 [45] and group 2 [47]) were given three acquisition trials, and one of them (group 1 ) was sleep deprived thereafter. On day 2, both groups were tested for memory (for temporal details, see protocol inset). On day 1 , both groups showed the same performance. On day 2 (bars), no difference $\left(P>0.05, \chi^{2}\right.$ test) was found between the two test groups, the sleep-deprived group $(\mathrm{N}=45)$ and the nonsleep-deprived group $(\mathrm{N}=47)$.

influence the decrease or increase in conditioned response. The results of this experiment not only document the specific effect of sleep deprivation on extinction memory consolidation but also rule out the possibility that shaking in itself might have a destructive effect on conditioned response, because the shaken animals showed higher response levels to the CS. From our experience, we know that stressed animals perform poorly on PER experiments. Our preliminary experiments (data not shown) showed that sleep-deprived bees learned as well as nonsleepdeprived bees during a three-trial acquisition learning protocol, while the more stressed bees performed poorly in the same task compared with nonstressed bees. Also, all sleep-deprived bees in
A

Group-1a and 2a: Sleep deprived Group-1b and 2b: Non-Sleep deprived

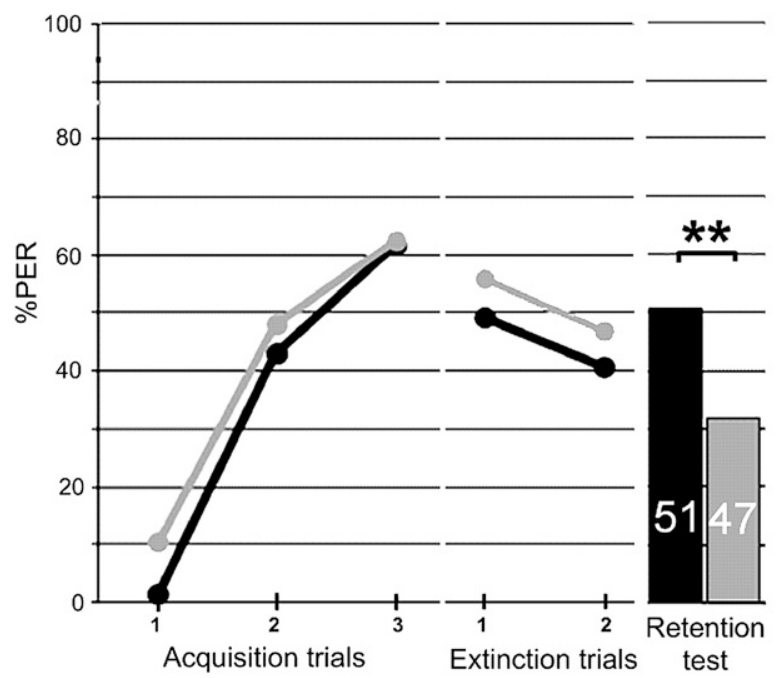

B

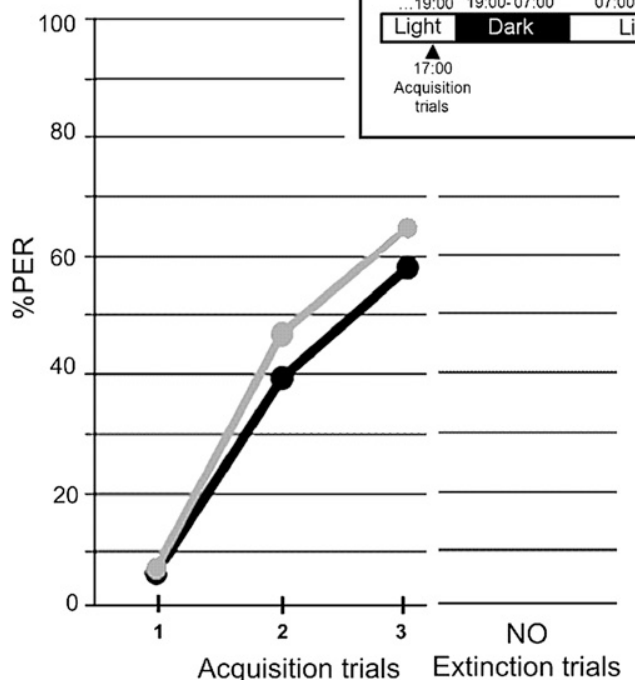

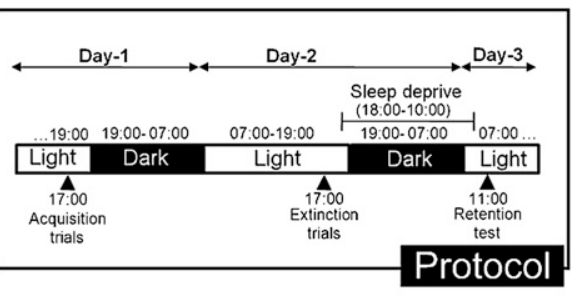

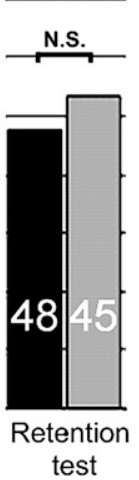

Figure 6. Sleep deprivation after extinction learning. ( $A$ ) On day 1 , two groups of bees (group 1a [51] and group 1b [47]) were given three acquisition trials. On day 2, both groups were given two extinction trials, and only group 1 a was sleep deprived thereafter. On day 3 , both groups were tested for memory. Both groups showed similar acquisition curves and extinction curves on days 1 and 2, respectively. On day 3, the sleep-deprived group 1a showed significantly higher response levels to the $\mathrm{CS}\left({ }^{* *} P<0.01, \chi^{2}\right.$ test) compared with group $1 \mathrm{~b}$, indicating that consolidation of extinction memory was compromised. (B) This experiment served as a control to the previous experiment. On day 1, two groups of bees (group 2a [48] and group 2b [45]) were given three acquisition trials. On day 2, bees were not given extinction trials and only group 2a was sleep deprived thereafter. On day 3 , both groups were tested for memory. Both groups showed similar acquisition curves on day 1 . On day 3 , sleep deprivation had no effect $\left(P>0.05, \chi^{2}\right.$ test) on retention. This indicates that the difference in retention scores found for groups $1 \mathrm{a}$ and $1 \mathrm{~b}$ on day 3 was not because of shaking per se but was specific for the consolidation of extinction memory. 
Figure 6 showed sleep rebound the following day, confirming the sleep deprivation effect. This shows that the effect we see is related to sleep rather than stress.

Our results therefore show that consolidation of acquisition memory is not changed by sleep deprivation but that consolidation of extinction memory is affected. Acquisition learning leads to a more robust memory than extinction learning because three-trial conditioning changed the conditioned response from $<10 \%$ to $>60 \%$ compared to a change of only $10 \%$ (from $\sim 55 \%$ to $\sim 45 \%)$ after a two-trial extinction learning. Thus, the consolidation of the weaker extinction memory could have been disrupted more easily by sleep deprivation, while the consolidation of the more robust acquisition memory may have not been disrupted by sleep deprivation. However, we cannot rule out the alternative possibility that the different kinds of memory rather than the strength of memory might be the important parameter. For instance, it could be that acquisition learning involves components that are more elementary than extinction learning, and such elementary forms of learning appear to require no contributions from the mushroom bodies (Scheiner et al. 2001; Malun et al. 2002). Experiments showed that ablating mushroom bodies did not affect acquisition learning, and it is proposed that such elemental learning might occur at the level of the antennal lobe (Giurfa 2003). If deprivation of sleep is more related to the function of the mushroom bodies (Joiner et al. 2006) than consolidation of memory after such configural forms as extinction, then learning may be more strongly affected. Furthermore, several studies have shown that acquisition learning and extinction learning are different in many aspects (Bouton et al. 1993; Myers and Davis 2002; Gottfried and Dolan 2004; Eisenhardt and Menzel 2007), and conditions have been reported that interfere with consolidation of extinction memory while sparing other forms of memory (Silvestri 2005; Fu et al. 2007; Dalton et al. 2008). It is thus possible that sleep deprivation affects only extinction learning, a question that needs to be addressed in further experiments. We, conclude that acquisition and extinction memories in honeybees might be mediated by different mechanisms, and sleep deprivation may help to uncover such differences.

\section{Materials and Methods}

Foraging honeybees (Apis mellifera carnica) were caught at the entrance of an outdoor (summer) or indoor (winter) hive $1 \mathrm{~d}$ prior to the experiment, immobilized on ice, and fixed inside plastic restraining tubes such that only the mandibles, proboscis, and antennae could move freely (Bitterman et al. 1983). The scapes of the antennae were attached to the head using low temperature melting wax (eicosane, Sigma-Aldrich) such that the flagellum was free to move. Bees were fed $30 \%$ sugar solution until satiation (about six to eight drops) and were kept under 12-h light and 12-h dark phase at about $25^{\circ} \mathrm{C}-27^{\circ} \mathrm{C}$.

Bees were checked for PER (unconditioned response [UR]) by lightly touching the antennae with $30 \%$ sucrose solution $10 \mathrm{~min}$ before training. Only bees that showed the UR were conditioned ( $<5 \%$ were discarded). One of three odors-2-octanol, limonene, and peppermint-was used as a CS, and 30\% sucrose solution was used as the unconditioned appetitive stimulus (US). The odors were delivered by an olfactometer (Galizia et al. 1997; Komischke et al. 2002). A continuous stream of air was blown over the bee's antennae, which was switched to a cartridge containing $4 \mu \mathrm{L}$ of odor pipetted onto a half square inch filter paper, inside a 1-mL syringe. For each experiment, four syringes were used: three syringes containing odors and one syringe containing odorless filter paper (air control). During training each bee was placed in front of the olfactometer with its antennae facing the airstream for $60 \mathrm{sec}$. During this period, either CS or US or both were presented to the bee, and an exhaust system behind removed the air.
For monitoring sleep, both antennae of the bees were video recorded. The bee was placed inside a cubical plastic box that had only one side open (Fig. 1A). A web camera (Philips ToUcam Pro II) was fixed on top of the box such that both the antennae of the bee were clearly visible as black structures against a white background. An infrared light was fixed alongside the camera for recording videos in the dark. Bees were kept under a 12-h dark (from 19:0007:00 h) phase followed by a 12-h light (from 07:00-19:00 h) phase. A bright-white light was kept in front of the open side of the box during the light phase. A temperature probe was positioned inside the box to monitor day and night temperatures, and a filter paper soaked in water kept the inside of the box humid. The web camera was connected to a personal computer via USB 2.0, and video software (Active webcam v6.2) was used to record nonstop videos for $19 \mathrm{~h}$ (the rest of the $5 \mathrm{~h}$ were used for preparation of bees) at five to 10 frames per second (fps). The videos were stored on hard-disk for online or offline analysis.

A custom-made program tracked and calculated the angular movements of the antennae (Fig. 1A). The user interactively defined the coordinates of the antennae on a video frame. Right and left antennal positions were defined by lines and arcs around each antenna, determining the area of movement of each antenna. The angles of the flagella were measured against an imaginary line between the two pedicles of the two antenna. After these coordinates were defined for one frame, the program automatically tracked the angular movements of each flagellum for all subsequent frames of the video. The data containing angles of the two flagella along with time of day were saved as a text file and analyzed online or offline using Matlab R14.

For sleep deprivation, bees were placed on a vortex (Fig. 1B) and shaken at $80-120 \mathrm{rpm}$ for $5 \mathrm{~min}$ and rested for $5 \mathrm{~min}$ during the entire light and dark phase.

For sleep rebound experiments, up to four animals (eight flagella) were simultaneously monitored using a web camera at 10 fps. Customized software was used to mask the regions of the camera image where the bees were located. The software then computed the pixel changes (mean intensity difference) of the antennal regions from one frame to another and reported it as absolute multiantennal activity. Since the lighting conditions may introduce changing amounts of noise, we calculate the relative multiantennal activity as the ratio between flagella activities versus background noise. The unit of multiantennal activity was pixel change per hour $(\Delta$ pixels $/ h)$. If the flagella did not move, then there was no pixel change and therefore relative multiantennal activity was 1 . The antennal activities of day 1 and day 3 were compared.

\section{Analysis}

Microsoft Excel, Matlab, and R-statistical software were used for tabulating and plotting results. To determine the normality of our data, Shapiro-Wilk and Anderson-Darling normality tests were used. Based on normality tests, paired $t$-tests, Wilcoxon's rank-sum test, and Wilcoxon's signed-rank test were applied for determining statistical significance; Bonferroni correction was applied for paired comparisons with $\alpha$ level (threshold $P$-value) set as 0.05 . For binomial data we used $\chi^{2}$ tests.

\section{References}

Andersen FO. 1968. Sleep in moths and its dependence on the frequency of stimulation in Anagasta kuehniella. Opusc Entomol 33: 15-24.

Bicker G, Menzel R. 1989. Chemical codes for the control of behaviour in arthropods. Nature 337: 33-39.

Bitterman ME, Menzel R, Fietz A, Schafer S. 1983. Classical conditioning of proboscis extension in honeybees (Apis mellifera). J Comp Psychol 97: 107-119.

Born J, Rasch B, Gais S. 2006. Sleep to remember. Neuroscientist 12: 410424 .

Bouton ME, Rosengard C, Achenbach GG, Peck CA, Brooks DC. 1993. Effects of contextual conditioning and unconditional stimulus presentation on performance in appetitive conditioning. QJExp Psychol $B$ 46: 63-95.

Bushey D, Huber R, Tononi G, Cirelli C. 2007. Drosophila hyperkinetic mutants have reduced sleep and impaired memory. J Neurosci 27: 5384-5393. 
Dalton GL, Wang YT, Floresco SB, Phillips AG. 2008. Disruption of AMPA receptor endocytosis impairs the extinction, but not acquisition of learned fear. Neuropsychopharmacology 33: 2416-2426.

Daurat A, Terrier P, Foret J, Tiberge M. 2007. Slow wave sleep and recollection in recognition memory. Conscious Cogn 16: 445-455.

Eban-Rothschild AD, Bloch G. 2008. Differences in the sleep architecture of forager and young honeybees (Apis mellifera). J Exp Biol 211: 2408-2416.

Eisenhardt D, Menzel R. 2007. Extinction learning, reconsolidation and the internal reinforcement hypothesis. Neurobiol Learn Mem 87: 167-173.

Fiebrig K. 1912. Schlafende insekten. Jenaische Zeit Naturwissenschaft 48: $315-364$.

Fu J, Li P, Ouyang X, Gu C, Song Z, Gao J, Han L, Feng S, Tian S, Hu B. 2007. Rapid eye movement sleep deprivation selectively impairs recall of fear extinction in hippocampus-independent tasks in rats. Neuroscience 144: 1186-1192.

Gais S, Lucas B, Born J. 2006. Sleep after learning aids memory recall. Learn Mem 13: 259-262.

Galizia CG, Joerges J, Kuttner A, Faber T, Menzel R. 1997. A semi-in-vivo preparation for optical recording of the insect brain. J Neurosci Methods 76: 61-69.

Ganguly-Fitzgerald I, Donlea J, Shaw PJ. 2006. Waking experience affects sleep need in Drosophila. Science 313: 1775-1781.

Giurfa M. 2003. Cognitive neuroethology: Dissecting non-elemental learning in a honeybee brain. Curr Opin Neurobiol 13: 726-735.

Gottfried JA, Dolan RJ. 2004. Human orbitofrontal cortex mediates extinction learning while accessing conditioned representations of value. Nat Neurosci 7: 1144-1152.

Greenspan RJ, Tononi G, Cirelli C, Shaw PJ. 2001. Sleep and the fruit fly. Trends Neurosci 24: $142-145$.

Hammer M, Menzel R. 1998. Multiple sites of associative odor learning as revealed by local brain microinjections of octopamine in honeybees. Learn Mem 5: 146-156.

Heisenberg M. 2003. Mushroom body memoir: From maps to models. Nat Rev Neurosci 4: 266-275.

Hendricks JC, Sehgal A, Pack AI. 2000. The need for a simple animal model to understand sleep. Prog Neurobiol 61: 339-351.

Hoffmann RW. 1937. Der insektenschlaf als reflektorische immobilisation. Naturwissenschaften 25: 359-366.

Joiner WJ, Crocker A, White BH, Sehgal A. 2006. Sleep in Drosophila is regulated by adult mushroom bodies. Nature 441: 757-760.

Kaiser W. 1988. Busy bees need rest, too: Behavioural and electromyographical sleep signs in honeybees. J Comp Physiol [A] 163: 565-584.

Kaiser W. 1995. Rest at night in some solitary bees-a comparison with the sleep-like state of honey bees. Apidologie (Celle) 26: 213-230.

Kaiser W, Steiner-Kaiser J. 1983. Neuronal correlates of sleep, wakefulness and arousal in a diurnal insect. Nature 301: 707-709.

Klein BA. 2003. Signatures of sleep in a paper wasp. Sleep 26: A115-A116.

Klein BA, Olzsowy KM, Klein A, Saunders KM, Seeley TD. 2008. Castedependent sleep of worker honey bees. J Exp Biol 211: 3028-3040.

Komischke B, Giurfa M, Lachnit H, Malun D. 2002. Successive olfactory reversal learning in honeybees. Learn Mem 9: 122-129.

Malun D, Giurfa M, Galizia CG, Plath N, Brandt R, Gerber B, Eisermann B. 2002. Hydroxyurea-induced partial mushroom body ablation does not affect acquisition and retention of olfactory differential conditioning in honeybees. J Neurobiol 53: 343-360.

Marshall L, Helgadottir H, Molle M, Born J. 2006. Boosting slow oscillations during sleep potentiates memory. Nature 444: $610-613$.

Menzel R. 2001. Searching for the memory trace in a mini-brain, the honeybee. Learn Mem 8: 53-62.
Menzel R, Giurfa M. 2001. Cognitive architecture of a mini-brain: The honeybee. Trends Cogn Sci 5: 62-71.

Myers KM, Davis M. 2002. Behavioral and neural analysis of extinction. Neuron 36: 567-584.

Pitman JL, McGill JJ, Keegan KP, Allada R. 2006. A dynamic role for the mushroom bodies in promoting sleep in Drosophila. Nature 441: 753756.

Pribbenow B, Erber J. 1996. Modulation of antennal scanning in the honeybee by sucrose stimuli, serotonin, and octopamine: Behavior and electrophysiology. Neurobiol Learn Mem 66: 109-120.

Ramon F, Hernandez-Falcon J, Nguyen B, Bullock TH. 2004. Slow wave sleep in crayfish. Proc Natl Acad Sci 101: 11857-11861.

Rasch B, Buchel C, Gais S, Born J. 2007. Odor cues during slow-wave sleep prompt declarative memory consolidation. Science 315: 1426-1429.

Robertson EM. 2009. From creation to consolidation: A novel framework for memory processing. PLoS Biol 7: 1000019. doi: 10.1371/journal. pbio. 1000019

Sauer S, Kinkelin M, Herrmann E, Kaiser W. 2003. The dynamics of sleeplike behaviour in honey bees. J Comp Physiol A Neuroethol Sens Neural Behav Physiol 189: 599-607.

Sauer S, Herrmann E, Kaiser W. 2004. Sleep deprivation in honey bees. J Sleep Res 13: 145-152.

Scheiner R, Weiß A, Malun D, Erber J. 2001. Learning in honey bees with brain lesions: How partial mushroom-body ablations affect sucrose responsiveness and tactile antennal learning. Anim Cogn 3: 227-235.

Schroter U, Malun D, Menzel R. 2007. Innervation pattern of suboesophageal ventral unpaired median neurones in the honeybee brain. Cell Tissue Res 327: 647-667.

Schuppe H. 1995. Rhythmic brain activity in sleeping bees. Wien Med Wochenschr 145: 463-464.

Schuppe H, Burrows M. 1998. Arousal shifts in quiescent locusts. J Exp Biol 201: $1719-1728$.

Seugnet L, Suzuki Y, Thimgan M, Donlea J, Gimbel SI, Gottschalk L, Duntley SP, Shaw PJ. 2009. Identifying sleep regulatory genes using a Drosophila model of insomnia. J Neurosci 29: 7148-7157.

Seymour JE, Carrette TJ, Sutherland PA. 2004. Do box jellyfish sleep at night? Med J Aust 181: 707.

Shaw PJ, Cirelli C, Greenspan RJ, Tononi G. 2000. Correlates of sleep and waking in Drosophila melanogaster. Science 287: 1834-1837.

Silvestri AJ. 2005. REM sleep deprivation affects extinction of cued but not contextual fear conditioning. Physiol Behav 84: 343-349.

Stephenson R, Chu KM, Lee J. 2007. Prolonged deprivation of sleep-like rest raises metabolic rate in the Pacific beetle cockroach, Diploptera punctata (Eschscholtz). J Exp Biol 210: 2540-2547.

Stollhoff N, Menzel R, Eisenhardt D. 2005. Spontaneous recovery from extinction depends on the reconsolidation of the acquisition memory in an appetitive learning paradigm in the honeybee (Apis mellifera). J Neurosci 25: 4485-4492.

Tobler I. 1983. Effect of forced locomotion on the rest-activity cycle of the cockroach. Behav Brain Res 8: 351-360.

Tobler II, Neuner-Jehle M. 1988. Rest in the scorpion-a sleep-like state? J Comp Physiol [A] 163: 227-235.

Wilhelm I, Diekelmann S, Born J. 2008. Sleep in children improves memory performance on declarative but not procedural tasks. Learn Mem 15: 373-377.

Received August 4, 2009; accepted in revised form August 26, 2009. 


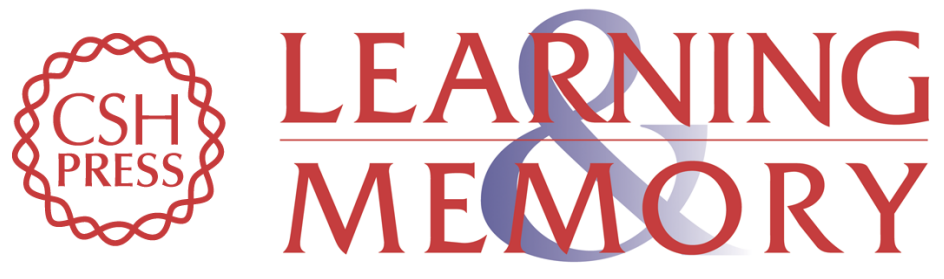

\section{Sleep deprivation affects extinction but not acquisition memory in honeybees}

Syed Abid Hussaini, Lisa Bogusch, Tim Landgraf, et al.

Learn. Mem. 2009, 16:

Access the most recent version at doi:10.1101//m.1578409

References

License

Email Alerting Service
This article cites 54 articles, 16 of which can be accessed free at: http://learnmem.cshlp.org/content/16/11/698.full.html\#ref-list-1

Receive free email alerts when new articles cite this article - sign up in the box at the top right corner of the article or click here. 